\title{
The Wnt/ $\beta$-catenin pathway regulates self-renewal of cancer stem-like cells in human gastric cancer
}

\author{
CHEN CAI and XIAOHAN ZHU \\ Department of Gastroenterology, The Second Xiangya Hospital, Central South University, \\ Changsha, Hunan 410011, P.R. China
}

Received December 15, 2011; Accepted February 15, 2012

DOI: $10.3892 / \mathrm{mmr} .2012 .802$

\begin{abstract}
Cancer stem cells (CSCs) possess the ability of self-renewal and tumor initiation. Targeting key signaling pathways that are active in CSC self-renewal is one approach to cancer therapy. Abnormal activation of the Wnt/ $\beta$-catenin pathway has been described in a wide variety of human cancers and in CSCs; however, the role of this pathway in gastric CSCs has not been reported. In our study, we investigated whether the $\mathrm{Wnt} / \beta$-catenin pathway plays an important role in gastric CSCs. First, we isolated cancer stem-like cells (CSLCs) from the human gastric cancer cell line MKN-45 using tumorsphere cultures. We tested whether tumorsphere cells were CSLCs using the following three criteria: i) We identified that the expression of the CSC marker CD44 was significantly greater in tumorsphere cells compared to adherent cells; ii) compared with adherent cells, the floating tumorsphere cells had greater self-renewing capacity; iii) in vivo xenograft studies showed that tumorsphere cells generate larger tumors than adherent cells at the same number. In addition, we studied the mechanism(s) by which the canonical Wnt signaling pathway acts in CSLCs. Western blotting and real-time PCR showed that the expression levels of $\beta$-catenin and c-myc, cyclin $\mathrm{d} 1$ and axin 2 were downregulated/upregulated with the inhibition/activation of the Wnt pathway. The pathway blocked by DKK-1 caused a higher reduction in the self-renewing capacity of MKN-45 tumorsphere cells and the pathway activated by lithium chloride improved the self-renewal of CSLCs. In conclusion, our data suggested that the $\mathrm{Wnt} / \beta$-catenin pathway is essential for the self-renewal of CSLCs in human gastric cancer.
\end{abstract}

Correspondence to: Dr Xiaohan Zhu, Department of Gastroenterology, The Second Xiangya Hospital, Central South University, Changsha, Hunan 410011, P.R. China

E-mail: xiaohanzhu668@gmail.com

Abbreviations: CSCs, cancer stem cells; CSLCs, cancer stem-like cells; LiCl, lithium chloride; LRP5/6, low-density lipoprotein receptor-related protein-5/6; TCF, T-cell factor; LEF, lymphoid enhancer factor

Key words: Wnt/ $\beta$-catenin, CSCs, gastric cancer, lithium chloride, DKK-1

\section{Introduction}

Tumor recurrence is one of the biggest problems in cancer therapy, and cancer stem cells (CSCs) are thought to be responsible for this. CSCs are a group of cells that have the ability of self-renewal and are tumor initiating. CSCs were first identified in human acute myeloid leukemia (1). Subsequent research has found that CSCs also exist in solid tumors, for instance in brain tumors (2), breast (3), colorectal (4) and liver cancer (5), carcinoma of cervix uteri (6), lung (7) and pancreatic cancer (8). Identification of molecular pathways essential for CSC self-renewal is critical for designing effective cancer therapeutics. The Wnt signaling pathway is an essential pathway that regulates non-neoplastic stem cells, including cell proliferation, differentiation and migration processes, which have been verified in various stem cells such as embryonic stem cells $(9,10)$, neural progenitor cells $(11,12)$, hematopoietic $(13)$ and cardiovascular stem cells (14). The similarities between normal adult stem cells and CSCs suggest that the signaling pathways involved in somatic stem cell maintenance may also be involved in the regulation of CSCs. This assumption is verified in certain CSCs, such as colon (15), prostate (16) and cutaneous cancer (17), chronic myeloid leukemia (18), acute myelogenous leukemia (19) and hepatic carcinoma (20). The Wnt signaling pathway is a highly conserved signaling pathway.

In the canonical Wnt signaling pathway, Wnt ligands bind to Frizzled-low-density lipoprotein receptor-related protein-5/6 (LRP5/6) receptor complexes and activate the cytoplasmic scaffold protein Dishevelled (DVL), resulting in the inhibition of $\beta$-catenin phosphorylation and degradation. $\beta$-catenin accumulates in the cytoplasm and translocates into the nucleus, activating the target genes associated with T-cell factor/lymphoid enhancer factor (TCF/LEF). DKK1, a member of the dickkopf family of secreted proteins, acts by directly binding to the Wnt co-receptor LRP5/6 and disrupts Wnt-induced canonical Wnt/ $\beta$-catenin pathway activation. Its inhibition effects on the Wnt signaling pathway, which regulates tumorigenesis, have been investigated in certain tumors: Zhu et al identified that DKK-1 secreted by mesenchymal stem cells was able to inhibit proliferation of human carcinoma cell lines through the canonical Wnt signaling pathway (21). Results of a previous study showed that DKK-1 acts as a tumor suppressor gene in colon cancer, with a higher secretion level following Wnt signaling pathway activation, which can be 
Table I. Primers used for real-time PCR.

\begin{tabular}{lll}
\hline Gene & \multicolumn{1}{c}{ Forward primer } & \multicolumn{1}{c}{ Reverse primer } \\
\hline Axin 2 & GAGTGGACTTGTGCCGACTTCA & GGTGGCTGGTGCAAAGACATAG \\
c-myc & GCAGCTGCTTAGACGCGCTGGA & CGCAGTAGAAATACGGCTGCAC \\
Cyclin 11 & ATGTTCGTGGCCTCTAAGATGA & CAGGTTCCACTTGAGCTTGTTC \\
GAPDH & GCACCGTCAAGGCTGAGAAC & TGGTGAAGACGCCAGTGGA \\
\hline
\end{tabular}

explained with a negative feedback loop (22). Lithium chloride ( $\mathrm{LiCl})$ activates the Wnt signaling pathway by its inhibition effect on GSK-3 $\beta$, which is part of the degradation complex.

In this study, we investigated whether the canonical Wnt signaling pathway activated or depressed by $\mathrm{LiCl} / \mathrm{DKK}-1$ produced any effect on the self-renewal of CSLs in the gastric cancer cell line MKN-45.

\section{Materials and methods}

Cell culture, formation of tumorspheres and sub-tumorspheres. The human gastric cancer cell line MKN-45 obtained from Central Experiment Labor of Xiangya was cultured in RPMI-1640 medium containing 10\% fetal bovine serum (FBS) and $1 \%$ antibiotic mixture. Cells were passaged every 2-3 days. Tumorspheres were derived by placing the MKN-45 cells into serum-free 1640 culture medium supplemented with human recombinant epidermal growth factor (EGF) (Peprotech, Rockville, NJ, USA) at a concentration of $20 \mathrm{ng} / \mathrm{ml}$, human recombinant basic fibroblast growth factor (bFGF) (Peprotech) at a concentration of $10 \mathrm{ng} / \mathrm{ml}, 1 \% \mathrm{~N}-2$ (Invitrogen, Carlsbad, CA, USA) and $1 \%$ antibiotic mixture, and the MKN-45 cells were plated in 6-well ultra-low attachment cell plates (Costar, Cambridge, MA, USA) at 1,000 cells per well. Ten days later, plates were analyzed for tumorsphere formation and were quantified using an inverted microscope at a magnification of x40 and x 100 .

Tumorspheres were dissociated at a density of 1,000 cells per $\mathrm{ml}$ and $100 \mu \mathrm{l}$ single cell suspension was seeded in each well of a 96-well plate in serum-free medium as described above. Each well was examined, and only the wells that contained a single cell were marked. Two weeks later, the wells were analyzed for sub-tumorsphere formation. Normal adherent cells were used as a control for their ability to form sub-tumorspheres. To test the effect of the $\mathrm{Wnt} / \beta$-catenin pathway on the formation of sub-tumorspheres, dissociated cells were treated with LiCl 10 mM (Sigma, St. Louis, MO, USA), DKK-1 200 ng/ $\mathrm{ml}$ (Peprotech) or control, phosphate-buffered saline (PBS), for $24 \mathrm{~h}$ and the formation of sub-tumorspheres was observed.

Western blot analysis. Tumorsphere cells or adherent cells were washed with ice-cold PBS and lysed directly in nuclear lysis buffer to collect whole cell extracts. The protein concentration of the total cell lysates was tested by an Enhanced BCA Protein assay kit (Beyotime, Institute of Biotechnology, Haimen, China). Protein samples for western blotting were prepared by boiling following the addition of denaturing sample buffer. Proteins were then separated using SDS-PAGE on a $10 \%$ gel, and transferred onto a nitrocellulose membrane by DYY-6C. Membranes were incubated at $4^{\circ} \mathrm{C}$ overnight with primary antibody, and subsequently incubated with secondary antibodies for $1 \mathrm{~h}$ at $37^{\circ} \mathrm{C}$. Protein bands were scanned using Genegnome Bio Imaging System (Syngene, Frederick, MD, USA) following the application of BeyoECL plus (Beyotime). The following concentrations were used for primary antibodies: CD44 antibodies 1:500 (Epitomics, Burlingame, CA, USA), $\beta$-catenin antibody 1:2000 (Epitomics) and $\beta$-actin antibody 1:500 (Santa Cruz Biotechnology, Santa Cruz, CA, USA).

Quantitative real-time PCR. Total RNA from the cell line was prepared with TRIzol (Takara) according to the manufacturer's instructions. The cDNA synthesis was carried out according to the protocol of the PrimeScript ${ }^{\circledR}$ RT Master Mix reagent kit (Takara DRR036S). The PCR reaction was performed with a Step OnePlus ${ }^{\mathrm{TM}}$ Real-time PCR System (Applied Biosystems, Carlsbad, CA, USA). The PCR primers were synthesized by BioSune Biotechnology (China), and the sequences $\left(5^{\prime}-3^{\prime}\right)$ are shown in Table I.

Xenograft model. An equal number $\left(1 \times 10^{6}\right)$ of freshly dissociated cells was suspended in $200 \mu \mathrm{l}$ PBS, and the tumorsphere cells were injected into the right underarm of each male nude mouse ( $n=6$ per group) and the same number of adherent cells was injected into the left underarm of each mouse. Thirty days later, mice were sacrificed by cervical dislocation and tumors were excised. Tumors were measured and then placed in $10 \%$ formalin for hematoxylin and eosin (H\&E) staining. Tumor size was calculated every 3 days using the formula: $2 \mathrm{x}$ larger diameter $\mathrm{x}$ (smaller diameter) ${ }^{2}$.

Hematoxylin and eosin. The excised tumors from nude mice were fixed in $10 \%$ formalin, embedded in paraffin and processed by standard histological methods. Tissue sections were then stained with $\mathrm{H} \& \mathrm{E}$ to assess morphology.

Statistical analysis. The experiments were repeated three times. Quantitative data were presented as the means \pm SD. The data were tested whether they were normally distributed or not. If data obeyed normal distribution, we analyzed the results using the Student's t-test; if data did not obey normal distribution, we analyzed the results using the Mann-Whitney U test with SPSS 18.0 statistical software. $\mathrm{P}<0.05$ was considered to indicate a statistically significant difference.

\section{Results}

Tumorsphere cells possessed the characteristics of cancer stem-like cells (CSLCs). MKN-45 adherent cells were grown 
A

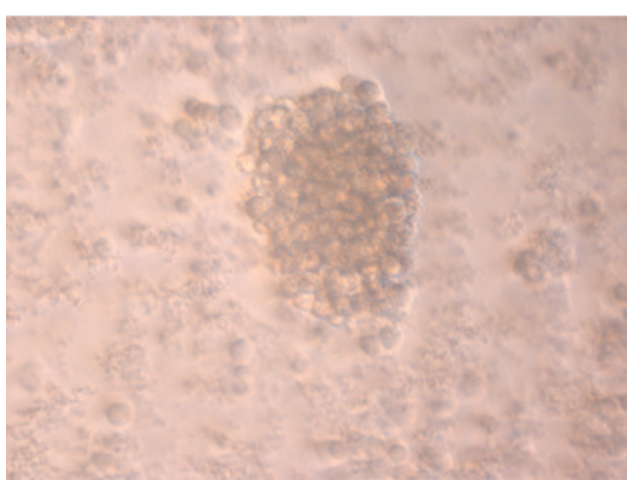

C

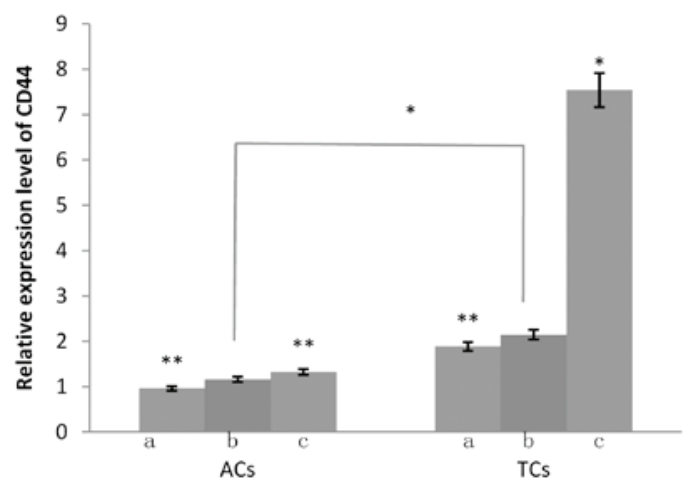

$\mathbf{E}$

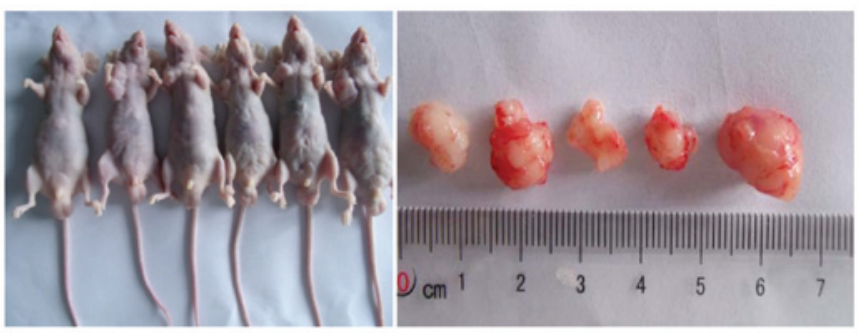

\section{Adherent cells Tumorsphere cells DKK-1 C LiCl DKK-1 C LiCl}
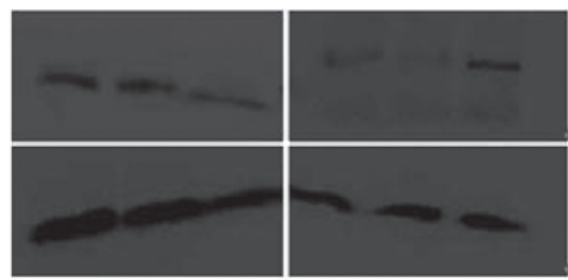

CD44

$\beta$-actin

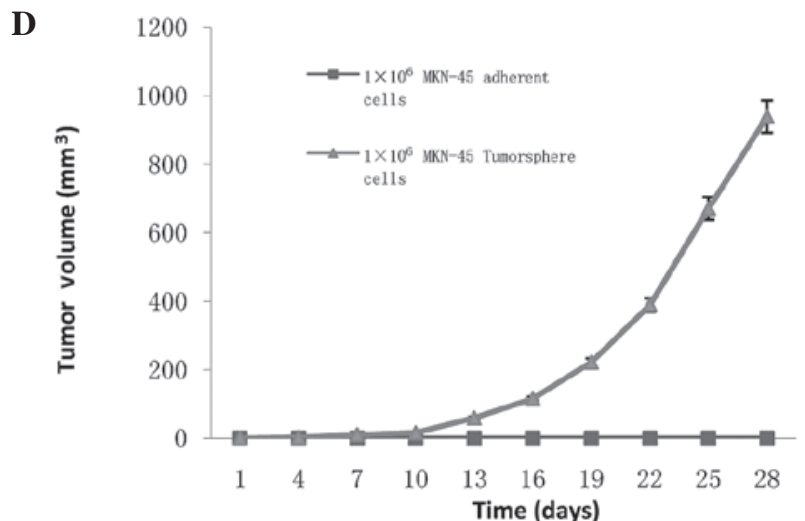

F

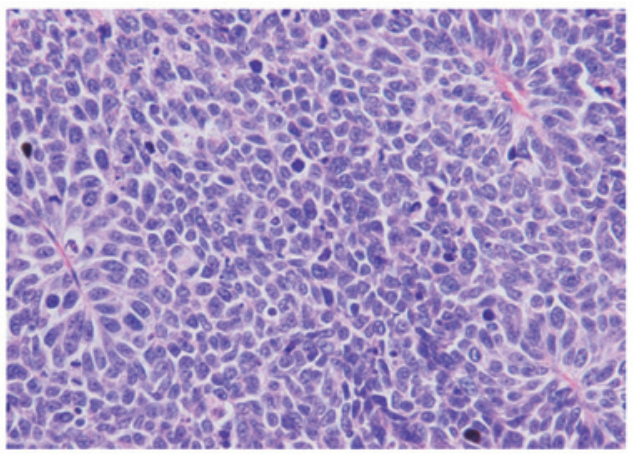

Figure 1. Tumorsphere cells possessed the characteristics of CSLCs. (A) Tumorspheres after 10 days of incubation in serum-free medium (original magnification, x600). (B) Western blot analysis of CD44 on adherent and tumorsphere cells following different treatments. (C) Relative expression level of CD44, regulated by $\beta$-actin. ${ }^{*} \mathrm{P}<0.05,{ }^{* *} \mathrm{P}>0.05$. (D) Subcutaneous tumor volume changes over time. (E) Subcutaneous tumor growth in nude mice following injection of the same number $\left(1 \times 10^{6}\right)$ of MKN-45 ACs to the left underarm and TCs to the right underarm. (F) H\&E staining analysis of xenografts derived from MKN-45 tumorsphere cells (original magnification, x400). ACs, adherent cells; TCs, tumorsphere cells; C, control; CSCLs, cancer stem-like cells; H\&E, hematoxylin and eosin; $\mathrm{LiCl}$, lithium chloride.

in serum-free medium as described in Materials and methods. After 10 days, tumorspheres consisting of approximately 50-100 cells were observed (Fig. 1A).

We examined the expression of CD44 in dissociated tumorsphere cells and adherent cells using western blotting. The results showed that the expression of CD44 was significantly greater in tumorsphere cells than in adherent cells (Fig. 1B and C).

The self-renewing capacity of these tumorspheres was assessed by dissociating them into single cells and growing them at a density of 1,000 cells per ml. After 2 weeks, single cells derived from MKN-45 tumorsphere cells generated sub-tumorspheres at a rate of $31.95 \%$ compared with $15.63 \%$ of adherent cells (Fig. 3A). This suggested that the self-renewing capacity of tumorsphere cells was significantly higher than that of adherent cells.

For xenograft studies, an equal number $\left(1 \times 10^{6}\right)$ of freshly dissociated tumorsphere cells or control adherent cells was injected into each mouse ( $n=6$ per group). The results showed that $83.3 \%(5 / 6)$ of the tumorsphere cells generated subcutaneous tumors, while no subcutaneous tumors were observed in the adherent cells whose activity was tested by trypan blue (Fig. 1D and E). This suggests that spheroid cells have a stronger tumorigenic capacity in nude mice than adherent cells.

The H\&E examination of xenografts derived from MKN-45 tumorsphere cells showed significant atypia and pleomorphism, and these tumors closely resembled the original human tumor, a poorly differentiated adenocarcinoma (Fig. 1F). 
These data suggest that tumorsphere cells represented CSLCs that had tumorigenic capacity.

$\beta$-catenin was more highly expressed in tumorsphere cells than in adherent cells, while no significant difference was detected in the pathway target genes. $\beta$-catenin is a key component of the $\mathrm{Wnt} / \beta$-catenin signaling pathway, and the level of $\beta$-catenin target genes represents the active state of the pathway. Therefore, we examined the expression levels of $\beta$-catenin by western blotting, and c-myc, cyclin $\mathrm{d} 1$ and axin 2 by quantitative real-time PCR.

Western blotting showed that the expression levels of $\beta$-catenin were higher in tumorsphere cells than in adherent cells (Fig. 2A). We used $\beta$-actin as a loading control, and the relative expression of $\beta$-catenin was found to be significantly higher in tumorsphere cells than in adherent cells (Fig. 2B), whereas the expression level of c-myc, cyclin $\mathrm{d} 1$ and axin 2 showed no significant difference in CSLCs and adherent cells, as shown by real-time PCR (Fig. 2C).

LiCl activates the Wnt/ $\beta$-catenin pathway, and activation of the pathway promotes the self-renewing capacity of $M K N-45$ tumorsphere cells. $\mathrm{LiCl}$ activates Wnt signaling by inhibiting GSK3 $\beta$, leading to $\beta$-catenin stabilization and translocation into the nucleus. Western blot analysis was used to investigate whether $\mathrm{LiCl}$ treatment upregulated the expression of $\beta$-catenin in MKN-45 cells. We found a marked increase of $\beta$-catenin in MKN-45 tumorsphere cells after stimulation with $\mathrm{LiCl}$ (Fig. 2A and B). To evaluate whether LiCl-mediated accumulation of $\beta$-catenin would lead to the activation of typical Wnt target genes, the mRNA expression level of c-myc, cyclin d1 and axin 2 was quantified by real-time PCR. Our results showed that the mRNA level of c-myc and cyclin d1 were significantly higher in MKN-45 tumorsphere cells which were treated with $\mathrm{LiCl}$ than those not treated with $\mathrm{LiCl}$ (Fig. 3B). Although the axin 2 mean expression level in tumorsphere cells treated with $\mathrm{LiCl}$ was higher than that in control cells, no statistical significance was observed (Fig. 3B). We also found an upregulation of the stem cell surface marker CD44 at the protein level by western blotting (Fig. $1 \mathrm{~B}$ and $\mathrm{C}$ ). $\mathrm{LiCl}$ treatment led to a significant enhancement in the capacity for the formation of sub-tumorspheres in MKN-45 tumorsphere cells (Fig. 3A), while no significant difference was observed in adherent cells with or without stimulation by $\mathrm{LiCl}$ (Figs. 1B and $\mathrm{C}, 2 \mathrm{~A}$ and $\mathrm{B}, 3 \mathrm{~A}$ and $\mathrm{C}$ ).

Taken together, these findings demonstrate that stimulation with $\mathrm{LiCl}$ may increase the self-renewal ability of CSLCs through activation of the Wnt/ $\beta$-catenin pathway. Additionally, it appears that only CSCs that have the ability of self-renewal and tumor initiation through abnormal activation of the Wnt/ $\beta$-catenin pathway; thus, no changes in adherent cells following treatment with the same pathway activator were observed.

DKK-1 blocks the Wnt/ $\beta$-catenin pathway and reduces the self-renewing capacity of $M K N-45$ tumorsphere cells. DKK-1 blocks the Wnt/ $\beta$-catenin pathway by binding to LRP5/6, preventing formation of the Frizzled-Wnt-LRP5/6 complex. Western blot analysis was used to investigate whether DKK-1 treatment was able to downregulate the expression

\section{A}

\section{Adherent cells Tumorsphere cells} DKK-1 C LiCl DKK-1 C LiCl

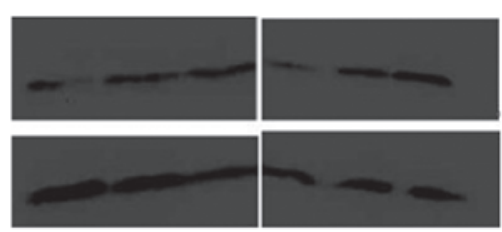

$\beta$-catenin

$\beta$-actin

B
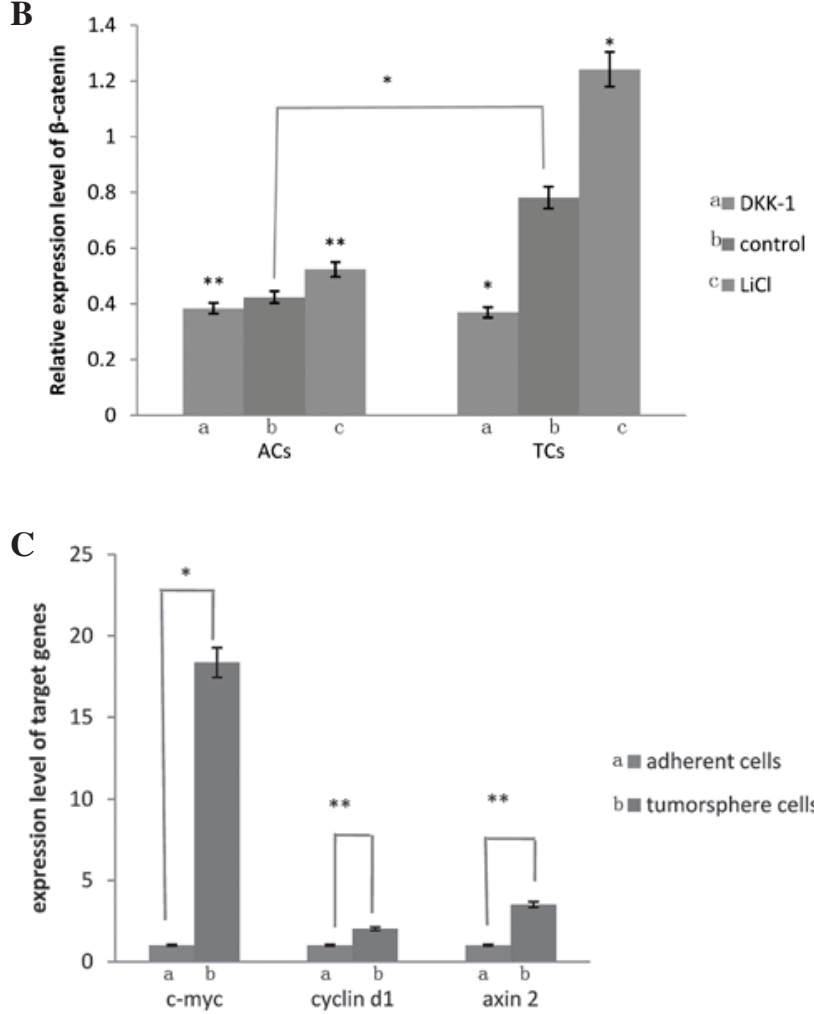

Figure 2. Wnt signaling in adherent and tumorsphere cells. (A) Western blot analysis of $\beta$-catenin on adherent and tumorsphere cells after different treatments. (B) Relative expression level of $\beta$-catenin, regulated by actin. " $\mathrm{P}<0.05$, ${ }^{* * *} \mathrm{P}>0.05$. (C) Expression levels of Wnt signaling target genes in adherent and tumorsphere cells. ACs, adherent cells; TCs, tumorsphere cells; C, control; $\mathrm{LiCl}$, lithium chloride.

of $\beta$-catenin. We found a marked decrease of $\beta$-catenin in MKN-45 tumorsphere cells following the treatment (Fig. 2A and B). To evaluate whether DKK-1-mediated degradation of $\beta$-catenin would lead to the inhibition of typical Wnt target genes, the mRNA expression level of c-myc, cyclin $\mathrm{d} 1$ and axin 2 was quantified by real-time PCR. The results showed that the mRNA level of these target genes was significantly lower in MKN-45 tumorsphere cells that were treated with DKK-1 than those without DKK-1 treatment (Fig. 3B). CD44 was not downregulated as expected (Fig. 1B and C). The reason for this may be that the cell surface marker was not specific enough. DKK-1 treatment led to a significant reduction in capacity for the formation of sub-tumorspheres in MKN-45 tumorsphere cells (Fig. 3A). However, no significant differences were observed in adherent cells with or without treatment with DKK-1 (Figs. 1B and C, 2A and B, 3A and C).

Taken together, these findings demonstrate that stimulation with DKK-1 could decrease the self-renewal ability of CSLCs 
A

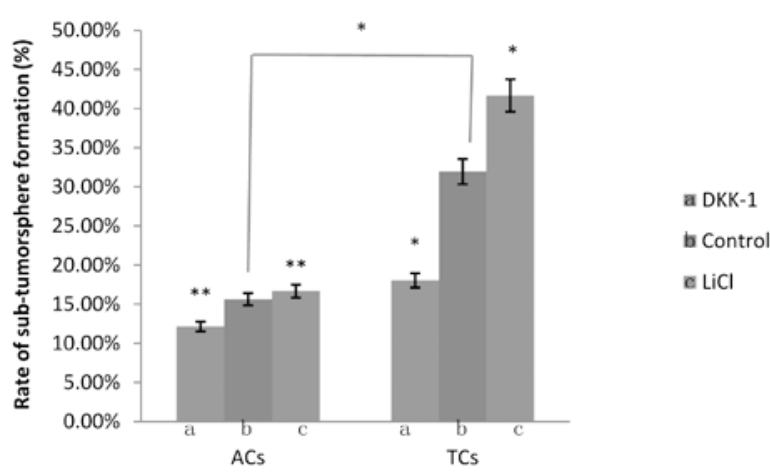

B
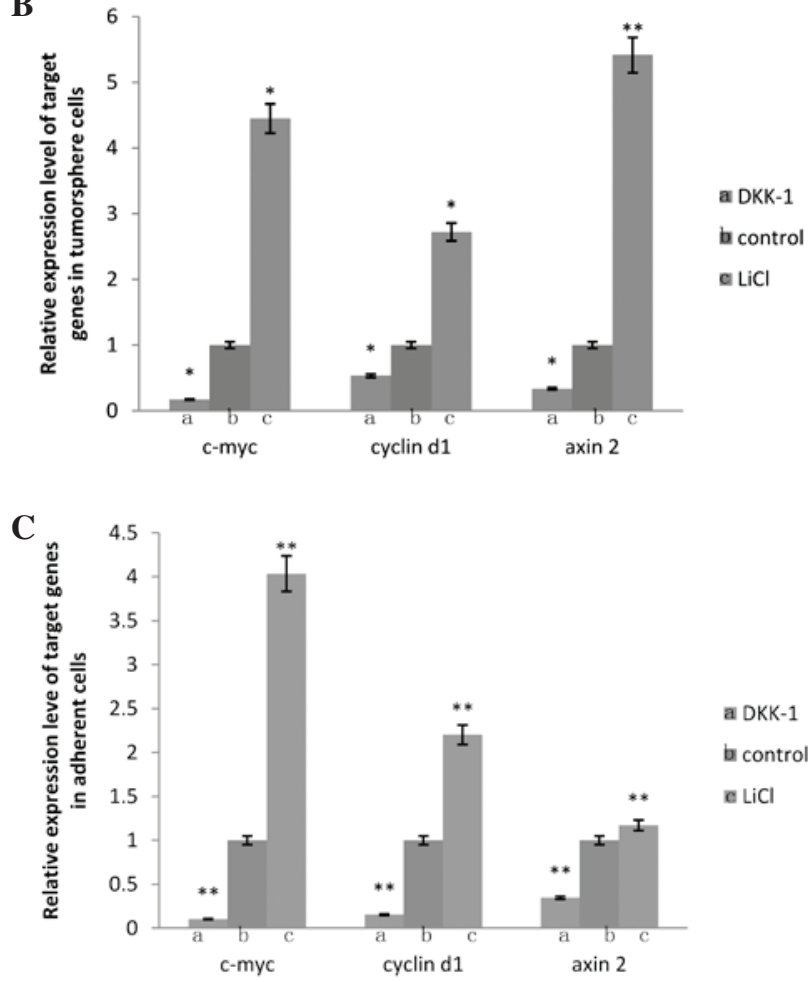

Figure 3. The effect of LiCl/DKK-1 on ACs and TCs. (A) The sub-tumorsphere formation ability of ACs and TCs under different conditions. (B) Expression level of Wnt signaling target genes in tumorsphere cells. (C) Expression level of Wnt signaling target genes in adherent cells. ${ }^{*} \mathrm{P}<0.05,{ }^{* *} \mathrm{P}>0.05$. ACs, adherent cells; TCs, tumorsphere cells; $\mathrm{LiCl}$, lithium chloride.

through inhibition of the Wnt/ $\beta$-catenin pathway. Moreover, it appears that only CSCs have the ability of self-renewal and can initiate tumors through abnormal regulation of the Wnt/ $\beta$-catenin pathway, which may explain the reason for the changes in adherent cells after treatment with the same pathway inhibitor not being detected.

\section{Discussion}

Despite the development of modern cancer therapy, cancer remains one of the major causes of mortality. CSCs are thought to be responsible for chemoresistance, metastasis and cancer recurrence. The elimination of CSCs may result in efficient cancer treatments. Signaling pathways, which regulate self-renewal of CSCs have been studied in a number of tumors, including the Wnt signaling pathway $(17,20)$, Hedgehog signaling pathway (23-25) and Notch signaling pathway (26). As for gastric cancer, for which the etiology is relatively clear, there is no research on the canonical Wnt signaling pathway regulating the self-renewal of its CSCs. Therefore, we performed a series of experiments to investigate whether or not this pathway is capable of regulating the self-renewal of gastric CSCs. The results showed that investigations should be conducted on how to effectively eliminate CSCs.

Spheroid colony formation in vitro, cell surface markers and xenotransplanted tumors in vivo are three approaches that have been used to identify CSCs. We used the tumorsphere culture method to isolate CSCs $(27,28)$. This is not only a way to obtain CSLCs, but also allows identification of the CSC characteristics of these cells. Using cell surface markers would be ideal for the isolation and identification of CSCs, if highly specific markers were identified. At present, markers that are not specific have been identified in various tumors (29-32). As for gastric cancer, Takaishi et al (27) found that $\mathrm{CD} 44^{+}$cells of gastric cancer behaved in the same manner as CSCs, thus CD44 may be regarded as a marker of gastric cancer stem cells. Our data have shown that tumorsphere cells express CD44 at significantly higher levels compared to adherent cells, and this expression identified CSC characteristics of the tumorsphere at the same time. We also used a tumorigenic assay, which showed that tumorsphere cells are more tumorigenic than adherent cells.

$\mathrm{C}$-myc, axin 2 and cyclin $\mathrm{d} 1$ are $\beta$-catenin target genes. The Wnt signaling pathway is involved in the regulation of certain types of CSCs (15-20). Additionally, the canonical Wnt signaling is activated in mammary mesenchymal subpopulation cells, but not in the parental cells, in an autocrine manner (33). Therefore, target genes should be elevated in CSCs compared to non-CSCs. Our data showed no significant difference in the expression level of Wnt signaling target genes between CSLs and adherent cells. This may be explained as follows: First, CSLCs are not equal to CSCs, and tumorspheres contained both CSCs and a small amount of non-CSCs, which explains the term CSLs. Similarly, there is also an extremely small number of CSCs present in adherent cells. Secondly, CSCs are quiescent and slow-cycling cells, and serum-free conditions are not ideal for CSC self-renewal. By contrast, the small number of CSCs in adherent cells exhibit renewal following culture in serum medium. Our data support this explanation. The Wnt signaling pathway is not maintained in non-CSCs, the number of CSCs in adherent cells is extremely small and this small group of CSCs is already in an active self-renewal situation. Therefore, our data showed that activation/inhibition of the Wnt signaling pathway had no significant effect on adherent cells. However, since Wnt signaling is maintained in the regulation of CSCs in the MKN-45 cell line and CSCs are enriched in the tumorsphere cells, our data have shown that activation of the Wnt pathway increases the expression level of target genes, with the exception of axin 2, and improves the ability of self-renewal in MKN-45 tumorsphere cells. By contrast, inhibition of the pathway decreases the expression level of target genes significantly and the self-renewal ability is reduced. Our data are in agreement with a former experiment: Yang et al (20) showed that following treatment with GSK-3 inhibitor, tumorigenic liver progenitor cells were increased 2 -fold to 11 -fold relative to the control group. Inhibition of the $\mathrm{Wnt} / \beta$-catenin pathway reduced the breast cancer stem cell 
population in vitro and in vivo (34). Axin 2, a direct target of the Wnt pathway, whose mRNA is expressed in a restricted pattern, is a negative regulator of the $\mathrm{Wnt} / \beta$-catenin pathway (35). Activation of the Wnt signaling pathway was expected to increase the expression level of target genes. However, no increase was found in the expression level of axin 2 following signal activation. The reason for this may be that axin 2 acts as a negative regulator of the $\mathrm{Wnt} / \beta$-catenin pathway, and a negative feedback loop inhibits the expression of axin 2 during the active course of the pathway. Our explanation has been tested previously: Axin 2 mRNA was not detected, whereas other Wnt/ $\beta$-catenin target genes were reactivated (36). Rough surface topography increases $\mathrm{Wnt} / \beta$-catenin target genes but decreases axin 2 expression without affecting $\beta$-catenin mRNA levels (37).

We found that the Wnt signaling pathway is important in the regulation of self-renewal of gastric CSCs, while it has no significant effects on non-CSCs. The combination of a powerful Wnt signaling inhibitor and chemotherapy generally used at present is likely to effectively control cancer. A number of small-molecule inhibitors or biological inhibitors (38) have been studied in the Wnt signaling pathway, but none are able to eliminate CSCs effectively. The combined blocking of multiple targets and pathways, which have evolved in the selfrenewal of CSCs, is required.

\section{Acknowledgements}

We thank MOE Key Lab of Environmental and Health, School of Public Health, Tongji Medical College, Huazhong University of Science and Technology for kindly providing an excellent experimental environment.

\section{References}

1. Lapidot T, Sirrd C, Vormoor J, et al: A cell initiatin human acute myeloid leukaemia after transplantation into SCID mice. Nature 367: 645-648, 1994

2. Singh SK, Clarke ID, Terasaki M, et al: Identification of a cancer stem cell in human brain tumors. Cancer Res 63: 5821-5828, 2003.

3. Al-Hajj M, Wicha MS, Benito-Hernandez A, et al: Prospective indentification of tumorigenic breast cancer cells. Proc Natl Acad Sci USA 100: 3983-3988, 2003.

4. Yeung TM and Mortensen NJ: Colorectal cancer stem cells. Dis Colon Rectum 52: 1788-1796, 2009.

5. Yang ZF, Ngai P, Ho DW, et al: Identification of local and circulating cancer stem cells in human liver cancer. Hepatology 47: 919-928, 2008

6. Feng D, Peng C, Li C, et al: Identification and characterization of cancer stem-like cells from primary carcinoma of the cervix uteri. Oncol Rep 22: 1129-1134, 2009.

7. Kim CF, Jackson EL, Woolfenden AE, et al: Identification of bronchioalveolar stem cells in normal lung and lung cancer. Cell 121: 823-835, 2005.

8. Li C, Heidt DG, Dalerba P, et al: Identification of pancreatic cancer stem cells. Cancer Res 67: 1030-1037, 2007.

9. Abu-Remaileh M, Gerson A, Farago M, et al: Oct-3/4 regulates stem cell identity and cell fate decisions by modulating Wnt/ $\beta$ catenin signaling. EMBO J 29: 3236-3248, 2010.

10. Paige SL, Osugi T, Afanasiev OK, et al: Endogenous Wnt/betacatenin signaling is required for cardiac differentiation in human embryonic stem cells. PLoS One 5: e11134, 2010.

11. Lange C, Mix E, Frahm J, et al: Small molecule GSK-3 inhibitors increase neurogenesis of human neural progenitor cells. Neurosci Lett 488: 36-40, 2011.

12. Zheng $\mathrm{H}$, Ying $\mathrm{H}$, Wiedemeyer $\mathrm{R}$, et al: PLAGL2 regulates Wnt signaling to impede differentiation in neural stem cells and gliomas. Cancer Cell 17: 497-509, 2010.
13. Reya T, Duncan AW, Ailles L, et al: A role for Wnt signaling in self-renewal of haematopoietic stem cells. Nature 423: 409-414, 2003.

14. Qyang Y, Martin-Puig S, Chiravuri M, et al: The renewal and differentiation of 1s11+ cardiocascular progenitors are controlled by a Wnt/beta-catenin pathway. Cell Stem Cell 1: 165-179, 2007.

15. Vermeulen L, De Sousa E Melo F, van der Heijden M, et al: Wnt activity defines colon cancer stem cells and is regulated by the microenvironmet. Nat Cell Biol 12: 468-476, 2010.

16. Bisson I and Prowse DM: WNT signaling regulates self-renewal and differentiation of prostate cancer cells with stem cell characteristics. Cell Res 19: 683-697, 2009.

17. Malanchi I, Peinado H, Kassen D, et al: Cutaneous cancer stem cell maintenance is dependent on beta-catenin signaling. Nature 452: 650-653, 2008.

18. Zhao C, Blum J, Chen A, et al: Loss of beta-catenin impairs the renewal of normal and CML stem cells in vivo. Cancer Cell 12: 528-541, 2007.

19. Wang Y, Krivtsov AV, Sinha AU, et al: The Wnt/beta-catenin pathway is required for the development of leukemia stem cells in AML. Science 327: 1650-1653, 2010.

20. Yang W, Yan HX, Chen L, et al: Wnt/beta-catenin signaling contributes to activation of normal and tumorigenic liver progenitor cells. Cancer Res 68: 4287-4295, 2008.

21. Zhu Y, Sun Z, Han Q, et al: Human mesenchymal stem cells inhibit cancer cell proliferation by secreting DKK-1. Leukemia 23: 925-933, 2009.

22. González-Sancho JM, Aguilera O, García JM, et al: The Wnt antagonist DICKKOPF-1 gene is a downstream target of betacatenin/TCF and is downregulated in human colon cancer. Oncogene 24: 1098-1103, 2005.

23. Zhao C, Chen A, Jamieson $\mathrm{CH}$, et al: Hedgehog signaling is essential for maintenance of cancer stem cells in myeloid leukaemia. Nature 458: 776-779, 2009.

24. Dierks C, Beigi R, Guo GR, et al: Expansion of Bcr-Abl-positive leukemic stem cells is dependent on Hedgehog pathway activation. Cancer Cell 14: 238-249, 2008.

25. Clement V, Sanchez P, de Tribolet N, et al: HEDGEHOG-GLI1 signaling regulates human glioma growth, cancer stem cell selfrenewal, and tumorigenicity. Curr Biol 17: 165-172, 2007.

26. Hoey T, Yen WC, Axelrod F, et al: DLL 4 blockade inhibits tumor growth and reduces tumor-initiating cell frequency. Cell Stem Cell 5: 168-177, 2009.

27. Takaishi S, Okumura T, Tu S, et al: Identification of gastric cancer stem cells using the cell surface marker CD44. Stem Cells 27: 1006-1020, 2009.

28. Goodrear SM, Amatangelo MD and Stearns ME: Dysplasia of human prostate CD133(hi) sub-population in NOD-SCIDS is blocked by c-myc anti-sense. Prostate 69: 689-698, 2009.

29. Tirino V, Desiderio V, Paino F, et al: Human primary bone sarcomas contain CD133+ cancer stem cells displaying high tumorigenicity in vivo. FASEB J 25: 2022-2030, 2011.

30. Haraguchi N, Ishii H, Mimori K, et al: CD13 is a therapeutic target in human liver cancer stem cells. J Clin Invest 20: 3326-3339, 2010.

31. Pang R, Law WL, Chu AC, et al: A subpopulation of CD 26+ cancer stem cells with metastatic capacity in human colorectal cancer. Cell Stem Cell 6: 603-615, 2010.

32. Gao MQ, Choi YP, Kang S, et al: CD24+ cells from hierarchically organized ovarian cancer are enriched in cancer stem cells. Oncogene 29: 2672-2680, 2010.

33. Scheel C, Eaton EN, Li SH, et al: Paracrine and autocrine signals induce and maintain mesenchymal and stem cell states in the breast. Cell 145: 926-940, 2011.

34. Li Y, Zhang T, Korkaya H, et al: Sulforaphane, a dietary component of broccoli/broccoli sprouts, inhitits breast cancer stem cells. Clin Cancer Res 16: 2580-2590, 2010.

35. Jho EH,Zhang T, Domon C, et al: Wnt/beta-catenin/Tcf signaling induces the transcription of Axin 2, a negative regulator of the signaling pathway. Mol Cell Biol 22: 1172-1183, 2002.

36. Brechbuhl HM, Ghosh M, Smith MK, et al: $\beta$-catenin dosage is a critical determinant of tracheal basal cell fate determination. Am J Pathol 179: 367-379, 2011.

37. Galli C, Passeri G, Ravanetti F, et al: Rough surface topography enhances the activation of Wnt/ $\beta$-catenin signaling in mesenchymal cells. J Biomed Mater Res A 95: 682-690, 2010.

38. Takahashi-Yanaga $\mathrm{F}$ and Kahn M: Targeting Wnt signaling: can we safely eradicate cancer stem cells? Clin Cancer Res 16: 3153-3162, 2010. 